\title{
A study of buckling phenomenon in dielectric elastomeric actuator with different potential energy functions
}

Deepak Kumar ( $\boldsymbol{\nabla}$ deepakkumaromre@manit.ac.in )

Maulana Azad National Institute of Technology Bhopal

Vinod Yadav

Maulana Azad National Institute of Technology Bhopal

Sankalp Gour

Maulana Azad National Institute of Technology Bhopal

Ayush Srivastav

Maulana Azad National Institute of Technology Bhopal

Research Article

Keywords: Continuum mechanics, Actuator

Posted Date: June 2nd, 2021

DOI: https://doi.org/10.21203/rs.3.rs-572274/v1

License: (c) (i) This work is licensed under a Creative Commons Attribution 4.0 International License.

Read Full License 


\title{
A study of buckling phenomenon in dielectric elastomeric actuator with different potential energy functions
}

\author{
Deepak Kumar, ${ }^{\text {a) }}$ Vinod Yadav, ${ }^{\text {b) Sankalp Gour, }}{ }^{\text {() }}$ and Ayush Srivastav ${ }^{\text {d) }}$ \\ Department of Mechanical Engineering, Maulana Azad National Institute of Technology Bhopal, Madhya-Pradesh, India-462003 \\ a)Corresponding author: deepakkumaromre@manit.ac.in \\ b) Electronic mail: vyadav@manit.ac.in \\ c) Electronic mail: sankalpgour@gmail.com \\ d) Electronic mail: as.181116002@manit.ac.in
}

\begin{abstract}
The present study deals with the buckling phenomenon modeling in a dome-shaped dielectric elastomeric (DE) actuator used in different aerodynamic and fluid power system applications. The DE actuator is a circular membrane type of actuator, which shows a large out-of-plane axial-symmetric deformation with an electrically induced loading condition. A classical continuum mechanics-based analytical model is developed to predict the electrically induced buckling deformation in the actuator. A detailed parametric study has been performed to see the influence of standard Neo-Hookean and Mooney-Rivlin types of potential energies on the geometrical and physical parameters. The findings show that the present model successfully links the sensitivity of different potential energies concerning the actuator's initial dome height and radius.
\end{abstract}

\section{INTRODUCTION}

Dielectric Elastomer (DE) is a particular class of non-conducting material with almost constant dielectric properties, usually incompressible and hyperelastic [1,2,3,4]. The commonly used examples of DE material for making smart actuators are polydimethylsiloxane (silicone) and VHB-4910 (very high bond). The DE-based system is coupled between two compliant electrodes such that it behaves like a parallel plate deformable capacitor unit. Electrostriction is the characteristic of DEs that causes them to deform their shape when subjected to an electric field. On applying an electrical voltage, they reduced in thickness and stretched in area. This phenomenon is known as electrically induced mechanical actuation. DE-based systems are the smart systems $[5,6,7,8]$ suited for the development of novel mechatronic actuators producing large strains. The current study focuses on a diaphragm (dome-shaped) DE actuator, a circular membrane type of actuator shown in Figure 1, offering a large out-of-plane axial-symmetric deformation with an electrically induced loading condition. The circular membrane is constrained here through a fixed, rigid ring to remain curved even during its inactive state. Potential applications of dome-shaped DE material-based spherical actuator are pumps, loud-speakers, electric generators, tactile displays, variable-curvature mirrors and tunable lenses etc.

In the literature, various researchers $[9,10,11]$ proposed the dome-shaped geometry of the actuator. They also discussed the actuator's operating principle that can work either in inflation mode [9, 10] or in buckled mode [11]. The actuator's buckled mode that is also focussed here does not require any membrane pre-stretch $[12,13]$ or external force than the inflation mode [14]. Goulbourne et al. [10] and He et al. [15] studied the out-of-plane electroelastic deformation of the dome-shaped actuator by solving the systems of non-linear differential equations. Specifically, Goulbourne et al. [10] solved one algebraic and two ordinary differential non-linear equations compared to the work of He et al. [15]. Later, Zhao and Suo [16] and O'Brien et al. [17] simulated the out-of-plane electroelastic deformation of the dome-shaped actuator using the finite element method. Recently, chang wt al. [18] fabricated spherical domeshaped pneumatic valves and performed a finite element method-based structural analysis. Their findings [18] favored the dome-shaped valve geometry over the typical rectangular cross-section. Alturki et al. [19] studied the snapthrough instability phenomenon for the shallow domes with cosine-curved profile and observed hysteretic and elastic energy dissipation in series combination. Further, Piranda and Bourgeois [20] developed a quasi-spherical microrobot, called catoms for Claytronics atoms could stick and move around each other. Furthermore, Tang et al. [21] silicon material-based dome-shaped transducer produced a sound pressure level as high as $88.21 \mathrm{~dB}$ in between 10 and $40 \mathrm{kHz}$.

From the literature, we may conclude that various modeling and simulational studies focusing on the out-of-plane electroelastic deformation of the dome-shaped actuator was performed. In the context of the current status of the problem, existing studies need a concise parametric study for the variation of the actuator's different parameters.

This paper performs an extensive parametric study to observe the influence of base radius values, initial dome height, initial thickness, and applied voltage on a cap tip deformation output of the dome-shaped actuator. Also, we 
develop a physics-based actuation force model in line with the literature to capture the performance of a dome-shaped DE actuator used in different aerodynamic and fluid power system applications. The formulated actuation force model connects the cap tip deformation (spherical shaped) output for different base radius values, initial dome height, and initial thickness with an applied electrical voltage difference.

\section{ELECTRICALLY INDUCED BUCKLING DEFORMATION MODEL}

In this section, a coupled nonlinear analytical model for a dome-shaped dielectric elastomeric (DE) actuator is presented in line with the literature $[10,14,15]$. The model is used to analyze a large out-of-plane axial-symmetric buckling deformation of the actuator for varying electrical voltage inputs.

The dome-shaped DE actuator is made of a thin-walled circular dielectric polymeric bladder-based membrane, as shown in Figure 1. The model is developed under the following assumptions:

1. The membrane material of the actuator is considered incompressible, isotropic, and hyperelastic.

2. The membrane is a perfect spherical cap with uniform thickness in both undeformed and deformed configurations.

3. The deformation is assumed to be axisymmetric.

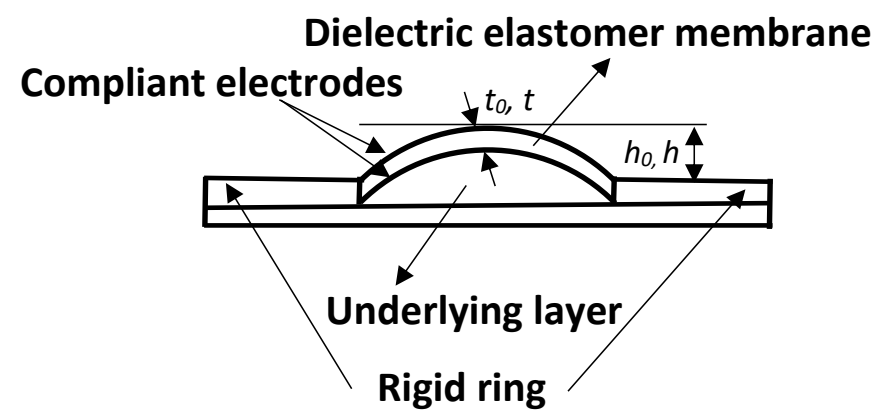

FIGURE 1: Schematic diagram of a buckling dielectric elastomer actuator

The geometry of the actuator in both undeformed and deformed configurations is described in Figure 1. The geometry of the actuator holds a variable surface area $A(h)=\pi\left(r^{2}+h^{2}\right)$ of any spherical cap with fixed base radius $r$ and with varying height $h$ for varying electrical voltage inputs. However, the volume of the actuator remains constant with the variation of a single longitudinal dimension and a fixed latitude dimension. Therefore, the principle stretches in principle directions for a given geometry of the actuator may be expressed as

$$
\lambda_{1}=\frac{r^{2}+h^{2}}{r^{2}+h_{0}^{2}}, \quad \lambda_{2}=1, \quad \lambda_{3}=\frac{1}{\lambda_{1} \lambda_{2}} \quad \text { (incompressibility) }
$$

where $h_{0}$ denotes the initial dome height of the actuator. The volume $V$ of the actuator is expressed as

$$
V=\pi\left(r^{2}+h_{0}^{2}\right) t_{0}=\pi\left(r^{2}+h^{2}\right) t
$$

As per the physical principle of the DE actuator, we may consider the DE membrane as a deformable parallel plate capacitor whose variable electrical capacitance $C(h)$ is defined as

$$
C(h)=\frac{\varepsilon_{r} \varepsilon_{0}\left(r^{2}+h^{2}\right)}{t}=\frac{\varepsilon_{r} \varepsilon_{0}\left(r^{2}+h^{2}\right) \lambda_{1}}{t_{0}},
$$


where $\varepsilon_{r}$ and $\varepsilon_{0}$ denote the dielectric constant of the material and the electrical permittivity of free space, respectively. On balancing the electromechanical energy for a dome-shaped like bubble deformation of the actuator in undeformed and deformed configurations, we may obtain an infinitesimal electrical work $d L_{\text {elec }}$ given as

$$
d L_{\text {elec }}=d W_{\text {mech }}+d W_{\text {elec }},
$$

where $d W_{\text {mech }}$ and $d W_{\text {elec }}$ denote the mechanical and electrical stores energies within the actuator system. An external energy supply source (i.e., a battery) is connected to provide the infinitesimal electrical work $d L_{\text {elec }}=\phi d q=\phi^{2} d C$ for the movement of an infinitesimal charge $d q$ from one compliant electrode to the other with an input voltage $\phi$. Thus, the capacitance of the system membrane changes by the amount $d C$ because of the electrically induced deformation. The mechanical $W_{\text {mech }}$ and electrical $W_{\text {elec }}$ energy expressions are obtained as

$$
W_{\text {mech }}(h)=V U_{\text {hyp }}\left(I_{1}, I_{2}, I_{3}\right), \quad W_{\text {elec }}(h)=\frac{1}{2} C \phi^{2},
$$

where $U_{\text {hyp }}\left(I_{1}, I_{2}, I_{3}\right)$ denote the potential energy density of an incompressible isotropic hyperelastic material of DE actuator as a function of the invariants $I_{1}, I_{2}$, and $I_{3}$. From the nonlinear theory of hyperelasticity, the invariants $I_{1}, I_{2}$, and $I_{3}$ may be expressed in terms of the principal stretches defined in equation (1) as

$$
I_{1}=\lambda_{1}{ }^{2}+\lambda_{2}{ }^{2}+\lambda_{3}{ }^{2}, \quad I_{2}=\frac{1}{\lambda_{1}{ }^{2}}+\frac{1}{\lambda_{2}{ }^{2}}+\frac{1}{\lambda_{3}{ }^{2}}, \quad I_{3}=\lambda_{1}{ }^{2} \lambda_{2}{ }^{2} \lambda_{3}{ }^{2} .
$$

There are various forms of potential energy density functions for an incompressible isotropic hyperelastic material of the DE actuator. In the current study, we use the standard Neo-Hookean and Mooney-Rivlin types of potential energy density functions given as

$$
U_{N H}=\mu\left(I_{1}-3\right), \quad U_{M R}=C_{1}\left(I_{1}-3\right)+C_{2}\left(I_{2}-3\right),
$$

where $\mu, C_{1}$ and $C_{2}$ are the constant parameters of the DE material of the actuator. On using the equations (3), (5) and (7), an explicit anaytical model to obtain the variable dome height $h$ of the DE actuator for the given electrical voltage $\phi$ and Neo-Hookean and Mooney-Rivlin potential energies material parameters $C_{1}$ and $C_{2}$ are expressed as

$$
h(M R)=\sqrt{\left(r^{2}+h_{0}^{2}\right)\left(1-\frac{\varepsilon_{r} \varepsilon_{0} \phi^{2}}{2\left(C_{1}+C_{2}\right) t_{0}^{2}}\right)^{-1 / 4}-r^{2}}, \quad h(N H)=\sqrt{\left(r^{2}+h_{0}^{2}\right)\left(1-\frac{\varepsilon_{r} \varepsilon_{0} \phi^{2}}{2 \mu t_{0}^{2}}\right)^{-1 / 4}-r^{2}} .
$$

The above relations represent a continuum mechanics-based analytical model to predict the electrically induced buckling deformation in the dome-shaped DE actuator.

\section{RESULTS AND DISCUSSION}

An extensive parametric study is carried out to obtain the cap tip deformation (spherical shaped) for different base radius values, initial dome height, and initial thickness process parameters of a dielectric elastomeric actuator $\left(r, t_{0}, h_{0}\right)$. Neo-Hookean (NH) and Monney-Rivlin (MR) strain energies are considered. The parametric study presented here observes the influence of $r, t_{0}$, and $h_{0}$ on a cap tip deformation. This cap tip deformation is estimated at different applied voltage, keeping other dielectric constant. To achieve a plane-axial symmetric deformation, a spherical shape of elastomer is considered. The material parameters for $\mathrm{NH}(\mu=11.12 \mathrm{kPa})$ and $\mathrm{MR}\left(C_{1}=11.13 \mathrm{kPa}, C_{2}=0.350\right.$ $\mathrm{kPa})$ are obtained by fitting the uniaxial stress-stretch experimental data [14] correspond to the silicone dielectric elastomer under uniaxial compression test using least-squire method.

Figure 2 shows the dependencies of cap tip deformation on radius $r$ at different dielectric constant $\varepsilon_{r}$ equals 4.6 and 7 using MR and NH strain energy density functions. The different values of $\varepsilon_{r}$ correspond to the different sets of material properties of the dielectric elastomeric actuators. It is observed that with increasing the voltage, the cap tip deformation increases. This effect is also more pronounced at a higher voltage. It is seen from Figure 2 that the cap tip deformation is higher when $\varepsilon_{r}$ is increased from 4.6 to 7 corresponds to the applied voltage. This is because the material properties are more pronounced towards the cap tip deformation while keeping the remaining process parameters of the actuators constant. To pick a range from 4.6 to 7 for dielectric constants, we may select the 
same silicon dielectric elastomer at very high restoring force frequencies. The electrons inside the dielectric material experience a restoring force produced by an applied electric field. It is also observed that as $r$ increased from 11.6 to $13.05 \mathrm{~mm}$, the cap tip deformation increased by $15.49 \%$ at $\varepsilon_{r}=4.6$ corresponding to the high voltage $12 \mathrm{kV}$ whereas $13.63 \%$ increased at $\varepsilon_{r}=7$. Similarly, when $\mathrm{r}$ is increased from 13.05 to $14.5 \mathrm{~mm}$, the cap tip deformation increased by $16.67 \%$ at $\varepsilon_{r}=4.6$ and by $15.2 \%$ at $\varepsilon_{r}=7$. The cap tip deformation is the same at low voltage with MR and NH strain energy functions. However, the results deviate at a high voltage within the variation of less than $1 \%$ error in both MR and NH strain energy functions.

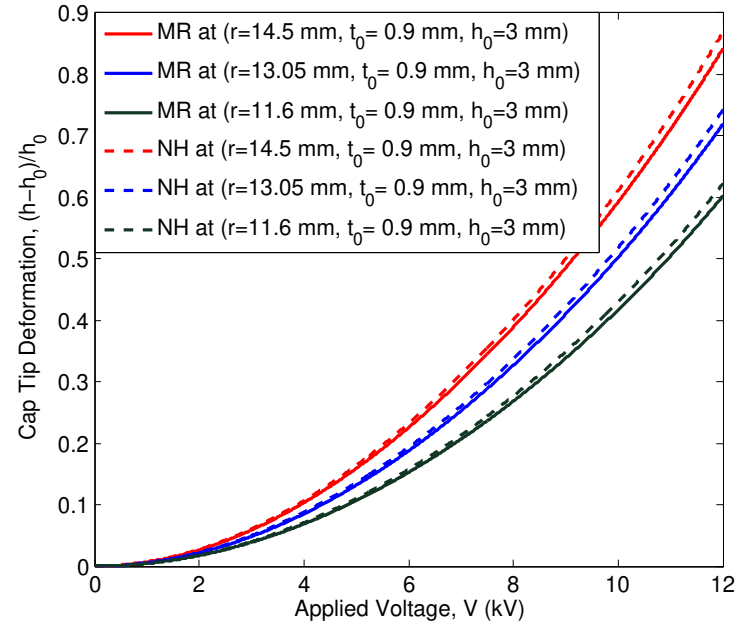

(a) $\varepsilon_{r}=4.6$

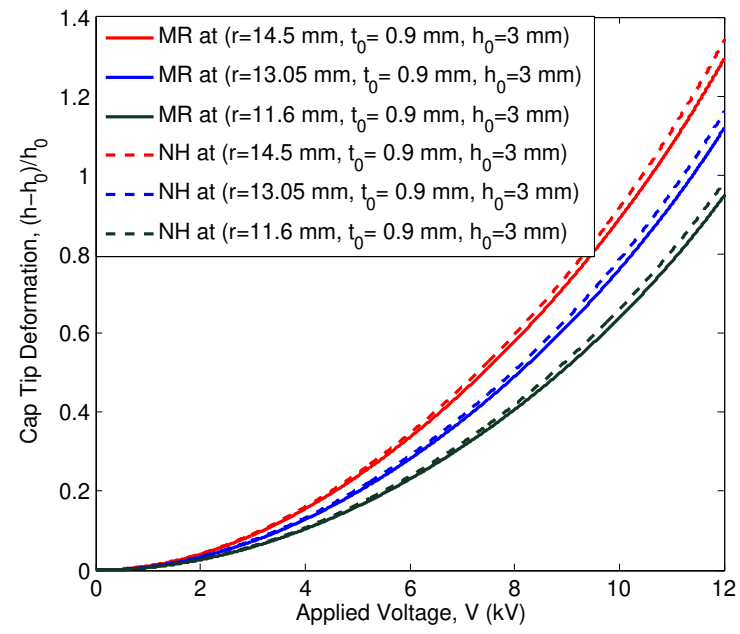

(b) $\varepsilon_{r}=7$

FIGURE 2: Effect of radius $r$ on $\varepsilon_{r}=4.6$ (a) and $\varepsilon_{r}=7$ (b) at different applied voltage

The influence of initial height $h_{0}$ on the cap tip deformation at different voltages is presented in Figure 3 . The variation of cap tip deformation with $h_{0}$ follows a different pattern than that for $r_{0}$. This can be ascertained by comparing Figure 2 and Figure 3. It is observed that with increasing the voltage, the cap tip deformation increases. Three different levels of $h_{0}$ with the variation of voltage are studied, and it can be seen from Figure 3 that the cap tip deformation pattern at $h_{0}$ equals 3 and $3.6 \mathrm{~mm}$ differs from that of $h_{0}=3.3 \mathrm{~mm}$. This may be due to the inconsistency of the spherical cap deformation's geometric parameters, and it can be further explored to understand the limitation of the deformation mechanism at $h_{0}=3.3 \mathrm{~mm}$. The rate of deformation is less sensitive, corresponding to the low voltage, and it becomes steeper with increasing the voltage at $h_{0}=3 \mathrm{~mm}$ and $h_{0}=3.6 \mathrm{~mm}$ (Figure 3 ). On the other hand the maximum cap tip deformation is up to 0.6 for $\varepsilon_{r}=4.6$ and $\varepsilon_{r}=7$. It is also observed that the variation of cap tip deformation with a voltage at different $h_{0}$ is consistent with MR as well as NH strain energy functions.

Figure 4 shows the dependencies of cap tip deformation on initial thickness $t_{0}$ at different dielectric constant $\varepsilon_{r}$ equals 4.6 and 7. Three different levels of initial thickness $0.72,0.81$, and $0.9 \mathrm{~mm}$ are considered. It is observed that cap tip deformation variations with the voltage at different $t_{0}$ follow a similar pattern with $r$. This can be ascertained by comparing Figure 2 and Figure 4. However, the cap tip deformation with the variations of $t_{0}$ is more pronounced than that of $r$. It is seen from Figure 4 that the cap tip deformation is increasing with changing the $\varepsilon_{r}$ from 4.6 to 7 . When $t_{0}$ is increased from 0.72 to $0.81 \mathrm{~mm}$, the cap tip deformation decreased by $22.22 \%$ and $26.02 \%$ at $\varepsilon_{r}=4.6$ and 7 , respectively for $\phi=12 \mathrm{kV}$. Similarly, when $t_{0}$ is increased from 0.81 to $9 \mathrm{~mm}$, the cap tip deformation decreased by $19.05 \%$ and $27.78 \%$ while $\varepsilon_{r}$ and $\phi$ constant.

Figure 5 shows the influence of cap tip deformation with the variations of $\varepsilon_{r}$ at different applied voltage $\phi$. Here, the interpretation of $\varepsilon_{r}$ refers to the different sets of dielectric elastomers-based actuators' material properties. It is observed that the pattern of cap tip deformation is linearly varied with $\varepsilon_{r}$ at three levels of $\phi$. However, the slope of the cap tip deformation pattern is changed. The slopes between the cap tip deformation and $\varepsilon_{r}$ are obtained as follows: 1,2 , and 3 correspond to applied voltage 3,6 , and $12 \mathrm{kV}$, respectively. It is also found that the cap tip deformation is similar at $\phi$ equals to 3 and $6 \mathrm{kV}$ for MR and NH strain energy functions. However, the MR and NH strain energy 


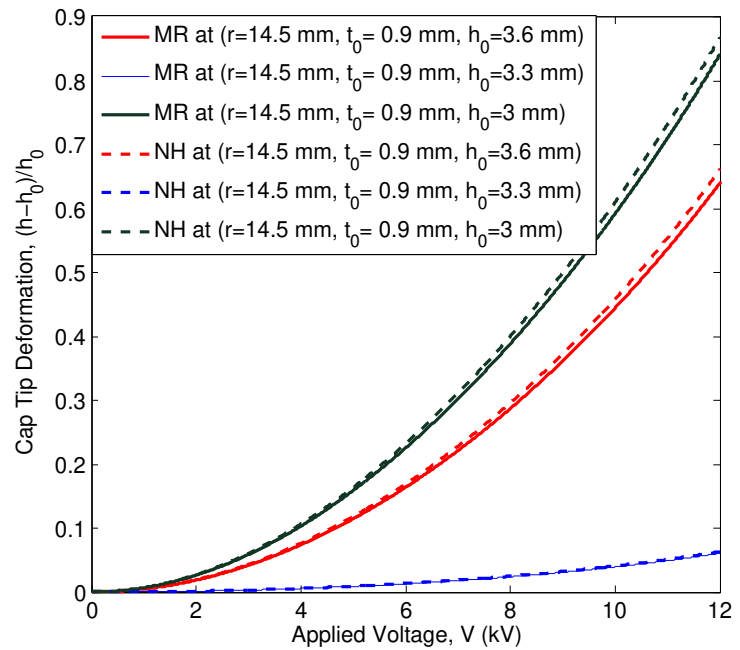

(a) $\varepsilon_{r}=4.6$

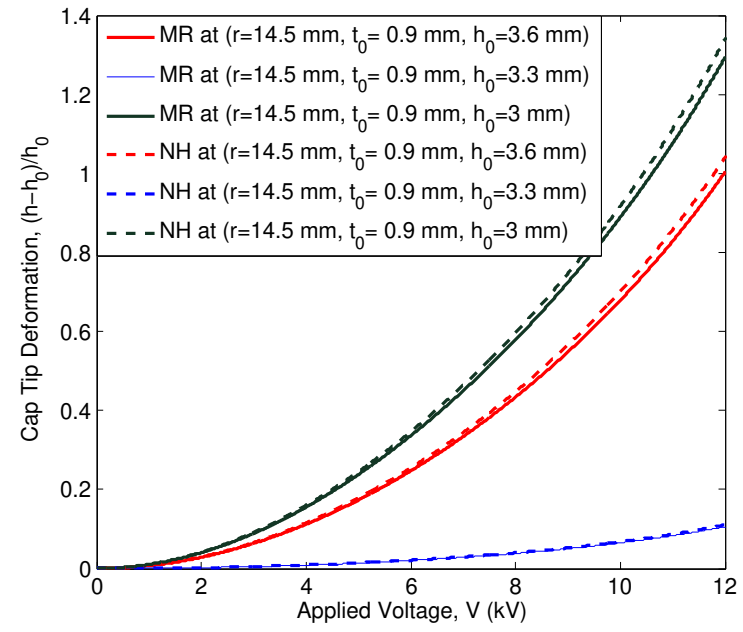

(b) $\varepsilon_{r}=7$

FIGURE 3: Effect of initial height $h_{0}$ Effect of radius $r$ on $\varepsilon_{r}=4.6$ (a) and $\varepsilon_{r}=7$ (b) at different applied voltage

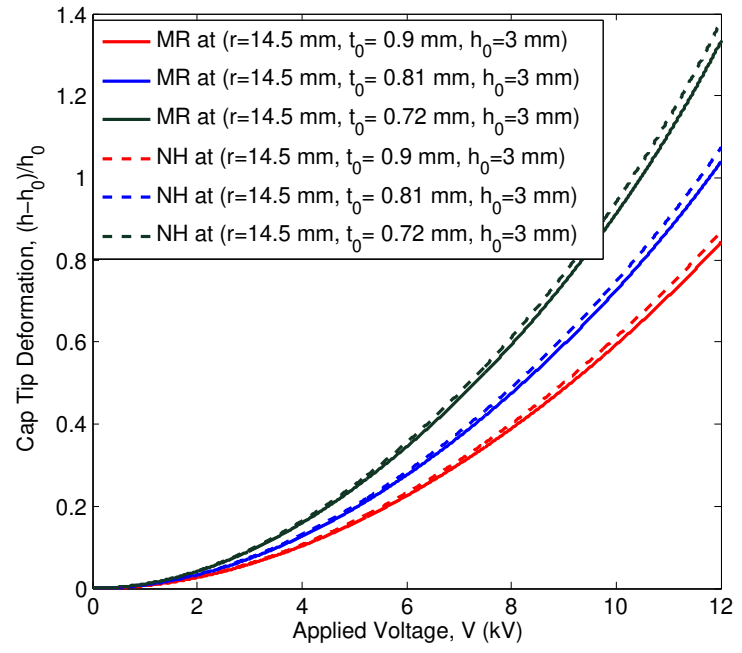

(a) $\varepsilon_{r}=4.6$

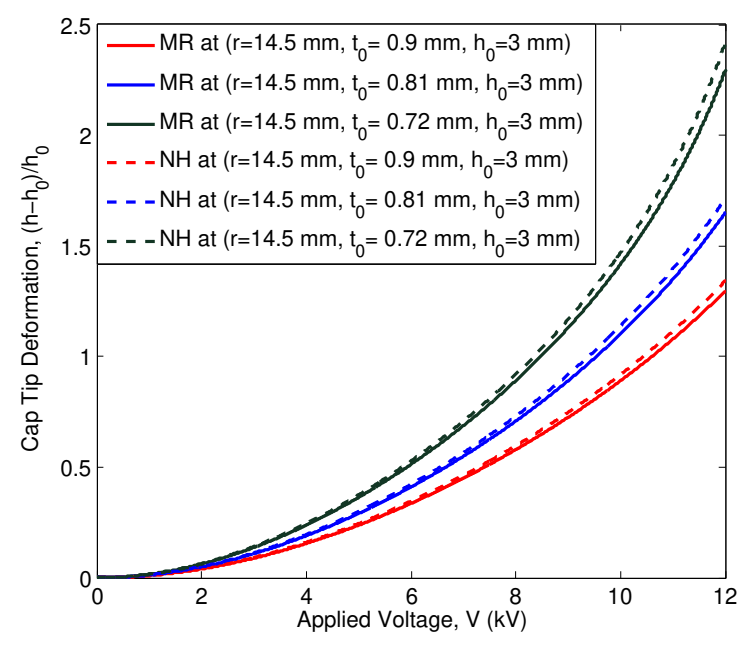

(b) $\varepsilon_{r}=7$

FIGURE 4: Effect of initial thickness $t_{0}$ Effect of radius $r$ on $\varepsilon_{r}=4.6$ (a) and $\varepsilon_{r}=7$ (b) at different applied voltage

functions' consistency becomes weaker at $V=12 \mathrm{kV}$ (Figure 5).

Based on the parametric study, it is observed that strain energy function (MR and NH are employed in the present paper) is not very sensitive at the low applied voltage for analyzing the cap tip deformation. The cap tip deformation is more pronounced with the variations of $r, h_{0}$, and $t_{0}$ at different applied voltage $\phi$. These observations can be useful to design and control the dielectric elastomers-based actuators and assess their applicability to fabrication and manufacturing. 


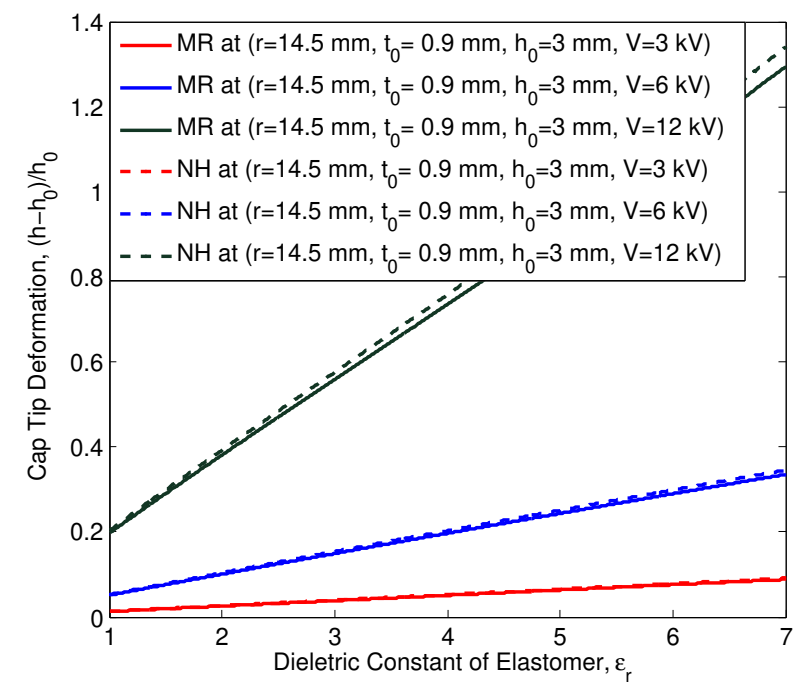

FIGURE 5: Effect of voltage $\phi$ at different dielectric constant of elastomers $\varepsilon_{r}$

\section{CONCLUDING REMARKS}

In the present work, an extensive parametric study has been performed to analyze the cap tip deformation of spherically/dome-shaped DE actuator by varying the base radius, initial dome height, and initial thickness with different applied voltage. Neo-Hookean and Monney-Rivlin strain energy functions are employed. We found that the cap tip deformation is highly sensitive at high applied voltage compared to the low applied voltage as expected from the previous studies. Hence, the paper results will be useful in the design and development of mechatronics based smart actuators. It is planned to simulate and demonstrate the experiments to realize the feasibility of the end product.

\section{ACKNOWLEDGMENTS}

The first author is gratefully acknowledging the support provided under the seed grant project entitled "Deformation Mechanics of Electro-magneto-active Polymers: Modeling with Experimental Validation" by Maulana Azad National Institute of Technology Bhopal, Madhya Pradesh, India-462003.

\section{REFERENCES}

1. R. E. Pelrine, R. D. Kornbluh, and J. P. Joseph, "Electrostriction of polymer dielectrics with compliant electrodes as a means of actuation," Sensors and Actuators A: Physical 64, 77-85 (1998).

2. S. Michel, X. Q. Zhang, M. Wissler, C. Löwe, and G. Kovacs, "A comparison between silicone and acrylic elastomers as dielectric materials in electroactive polymer actuators," Polymer international 59, 391-399 (2010).

3. D. Kumar and S. Sarangi, "Electro-magnetostriction under large deformation: Modeling with experimental validation," Mechanics of Materials 128, 1-10 (2019).

4. D. Kumar and S. Sarangi, "Electro-mechanical instability modelling in elastomeric actuators: A second law of thermodynamics-based approach," Soft Materials 17, 308-320 (2019).

5. D. Kumar, S. Ghosh, S. Roy, and S. Santapuri, "Modeling and analysis of an electro-pneumatic braided muscle actuator," Journal of Intelligent Material Systems and Structures, 1045389X20953624 (2020).

6. D. Kumar, S. K. Behera, and S. Sarangi, "Finite deformation of a dielectric cylindrical actuator: A continuum mechanics approach," in Recent Advances in Computational Mechanics and Simulations (Springer, 2020) pp. 401-417.

7. D. Kumar, S. Sarangi, and P. Saxena, "Universal relations in coupled electro-magneto-elasticity," Mechanics of Materials 143, 103308 (2020).

8. D. Kumar and S. Sarangi, "Instability analysis of an electro-magneto-elastic actuator: A continuum mechanics approach," AIP Advances $\mathbf{8}$, 115314 (2018). 
9. R. E. Pelrine and R. D. Kornbluh, "Electroactive polymer devices," (2003), uS Patent 6,545,384.

10. N. Goulbourne, E. Mockensturm, and M. Frecker, "Electro-elastomers: large deformation analysis of silicone membranes," International Journal of Solids and Structures 44, 2609-2626 (2007).

11. F. Carpi, G. Fantoni, G. Frediani, and D. De Rossi, "Buckling actuators with integrated displacement sensor," Dielectric elastomers as electromechanical transducers: Fundamentals, materials, devices, models and applications of an emerging electroactive polymer technology (2008).

12. D. Kumar and S. Sarangi, "Pre-stretching effect modeling of a dielectric elastomer under the electrical loading condition," Materials Today: Proceedings 24, 290-297 (2020).

13. D. Kumar, K. Arya, and S. Sarangi, "Modeling of a pre-stretched dielectric elastomer: A second law of thermodynamics-based approach," in Journal of Physics: Conference Series, Vol. 1276 (IOP Publishing, 2019) p. 012047.

14. R. Vertechy, A. Frisoli, M. Bergamasco, F. Carpi, G. Frediani, and D. De Rossi, "Modeling and experimental validation of buckling dielectric elastomer actuators," Smart Materials and Structures 21, 094005 (2012).

15. T. He, X. Zhao, and Z. Suo, "Dielectric elastomer membranes undergoing inhomogeneous deformation," Journal of Applied Physics 106 $083522(2009)$.

16. X. Zhao and Z. Suo, "Method to analyze programmable deformation of dielectric elastomer layers," Applied Physics Letters 93, 251902 (2008).

17. B. O'Brien, T. McKay, E. Calius, S. Xie, and I. Anderson, "Finite element modelling of dielectric elastomer minimum energy structures," Applied Physics A 94, 507-514 (2009).

18. C. K. Oh, S. W. Lee, and O. C. Jeong, "Fabrication of pneumatic valves with spherical dome-shape fluid chambers," Microfluidics and Nanofluidics 19, 1091-1099 (2015).

19. M. Alturki and R. Burgueño, "Response characterization of multistable shallow domes with cosine-curved profile," Thin-Walled Structures 140, 74-84 (2019).

20. B. Piranda and J. Bourgeois, "Geometrical study of a quasi-spherical module for building programmable matter," in Distributed Autonomous Robotic Systems (Springer, 2018) pp. 387-400.

21. Y. Tang, Y. Wang, L. Zhao, A. Shkel, and E. S. Kim, "Micromachined piezoelectric ultrasonic transducer based on dome-shaped diaphragm supported by flat square diaphragm," in 2016 IEEE 29th International Conference on Micro Electro Mechanical Systems (MEMS) (IEEE, 2016) pp. 1110-1111. 